\title{
Composition des lipides de l'œuf chez des poules Leghorn normales et naines
}

\author{
Y. DEMARNE, P. MERAT * et Andrée PIHET \\ I.N.R.A., Station de Recherches de Nutrition \\ * Laboratoire de Génétique factorielle, Centre de recherches zootechniques, \\ F 78350 Jouy-en-Josas
}

\begin{abstract}
Résumé
Dans une population Leghorn blanche en ségrégation pour le gène de nanisme lié au sexe $d w$, des oeufs (un par poule) provenant de 18 couples de sceurs, l'une de taille normale, l'autre naine, ont été analysés pour la teneur du jaune en lipides totaux, triglycérides, phospholipides et cholestérol, ainsi que pour la composition en acides gras des lipides totaux. En outre, pour les cufs provenant de 6 couples, la composition en acides gras était déterminée d'une part pour les triglycérides, d'autre part pour les phospholipides.

$\mathrm{Ni}$ la teneur du jaune en lipides totaux, ni la répartition de ces lipides en triglycérides, phospholipides et cholestérol ne diffèrent significativement suivant le génotype au locus Dw. Par contre, la composition en acides gras des lipides totaux et des triglycérides manifeste des différences hautement significatives selon ce génotype. En particulier on observe une teneur des jaunes plus faible en acide oléique et plus élevée en acide linoléique pour le génotype dw comparé au génotype $\mathrm{Dw}^{+}$. L'hypothèse est émise selon laquelle ces différences pourraient être le reflet d'une participation accrue des lipides alimentaires à la synthèse des triglycérides vitellins chez la poule naine.
\end{abstract}

Mots clés: Volaille, nanisme, auf, lipides, acides gras.

\section{Summary}

The composition of yolk lipids in eggs of normal and dwarf Leghorn hens

The composition of yolk lipids has been studied in à White Leghorn strain segregating for the sex-linked dwarf gene $d w$ using 18 pairs of full-sisters, one normal-sized and the other dwarf. The percentages of total lipids, triglycerides, phospholipids and cholesterol and the fatty acid composition of the total lipids were determined in the egg yolks of these hens (one egg per hen). In addition, the fatty acid composition of the triglycerides and phospholipids was evaluated in the eggs of 6 pairs.

Neither the total lipids in the yolk nor the proportion of triglycerides, phospholipids and cholesterol in the total lipids differed significantly according to the genotype at the Dw locus. Conversely, the fatty acid composition of the total lipids and of triglycerides was significantly different in $\mathrm{Dw}+$ and dw genotypes : there was a lower percentage of oleic acid and higher percentage of linoleic acid in the yolk of the dw genotype. It is hypothesized that these differences might reflect increased participation of dietary lipids in triglyceride synthesis in the vitellus of dwarf hens.

Key words : Poultry, sex-linked dwarfism, egg, lipid, fatty acids. 


\section{Introduction}

Les effets physiologiques associés au gène de nanisme lié au sexe dw chez la poule ont été passés en revue, en particulier par Guilladume (1976). Parmi ces effets, une influence sur le métabolisme lipidique est à remarquer. Les poulets nains ont une teneur en lipides corporels plus élevée que des poulets de taille normale (MERAT \& Guillaume, 1969 ; Guillaume, 1969 ; Ricard, 1970 ; Ouhayoun, 1970 ; PolkinGHORNE, 1974). Guillaume (1975) note que, chez les poulets comme chez les poules en ponte, la concentration du sérum en acides gras libres est abaissée par le gène dw. D'autre part, TouchBuRn \& Blum (1972), TouchBuRn et al. (1975) sur de jeunes poulets, puis Calabotta et al. (1982) sur coqs adultes, concluent que le gène de nanisme lié au sexe stimule l'anabolisme des lipides mais réduit la lipolyse.

Chez la poule en ponte, les phénomènes sont compliqués par la vitellogenèse. Celle-ci apparaît, dans l'ensemble, réduite chez les poules dw (Guillaume, 1976). Le nombre de follicules en phase de grand accroissement à un moment donné sur la grappe ovarienne est plus faible en moyenne pour les poules naines que pour les normales, dans une souche lourde (JAAP \& MoHAmmadian, 1969) ou dans une population légère de type Leghorn (BANERJEE, MERAT \& BORDAS, 1982). On observe une nette réduction de la longueur des séries de ponte chez la poule naine (MERAT, 1972). Enfin, Merat \& Ricard (1974) observent que le pourcentage de graisse abdominale des pondeuses naines, en corrélation positive avec le pourcentage de lipides corporels totaux, est inférieur à celui des poules de taille normale de même origine, contrairement à ce que l'on observe dans le jeune âge.

A notre connaissance, ces indications globales d'un métabolisme lipidique modifié par le gène $\mathrm{dw}$, notamment chez la poule pondeuse, n'ont pas été complétées par une étude comparée de la composition des lipides de l'œuf. Des recherches dans ce sens peuvent conduire à une meilleure compréhension des effets du gène en cause, et donner des indications, par exemple, sur la composition lipidique optimum des rations alimentaires. De plus, on connaît peu d'effets génétiques sur la composition en acides gras des tissus ou des produits (pour l'œuf, voir revue par WASHBURN, 1979).

Tel est l'objet de cette première étude, essentiellement descriptive, portant sur des œufs de sœurs normales et naines recevant ad libitum un régime alimentaire identique.

\section{Matériel et méthodes}

\section{A. Génotypes comparés, âge, conditions d'élevage}

Les œufs analysés provenaient de poules d'une population de type Leghorn blanche, dans laquelle le gène de nanisme lié au sexe était maintenu en ségrégation, par l'accouplement de coqs hétérozygotes $\mathrm{Dw}^{+} \mathrm{dw}$ avec des poules $\mathrm{dw}$. Parmi les filles obtenues, écloses en septembre 1980 , des couples de sœurs, l'une normale $\left(\mathrm{Dw}^{+}\right), \mathrm{l}^{\prime}$ au- 
tre naine (dw) étaient choisis. Un æuf était prélevé au même âge pour chaque poule. Ces poules faisaient partie du «lot témoin» décrit dans BANERJEE et al. (1982).

Dans une première série, les aufs de 6 couples de pondeuses âgées d'environ 13 mois étaient prélevés pour la détermination de la composition en acides gras des lipides totaux du jaune.

Une seconde série comprenait les œufs de 12 couples de poules du même troupeau, âgées de 18 mois environ. Sur tous les œufs étaient déterminés le poids de l'œuf, le poids du jaune, sa teneur en lipides totaux, triglycérides, phospholipides et cholestérol, ainsi que la composition en acides gras des lipides totaux.

Enfin, dans la même série, sur 6 des couples précédents, la composition en acides gras était déterminée d'une part pour les triglycérides, et d'autre part pour les phospholipides.

Les poules étaient contrôlées en cages individuelles. Elles recevaient toutes ad libitum le même aliment à environ 16 p. 100 de protéines totales et $2600 \mathrm{Kcal} / \mathrm{kg}$ d'énergie métabolisable. La composition est donnée au tableau 1.

TABLEAU 1

Composition de l'aliment.

Composition of the feed.

\begin{tabular}{|c|c|}
\hline Composant & Pourcentage \\
\hline Blé & 21,5 \\
\hline Maïs $\ldots \ldots \ldots \ldots \ldots \ldots \ldots \ldots \ldots \ldots \ldots \ldots$ & 45 \\
\hline Tourteau de soja extraction & 6 \\
\hline Tourteau de tournesol extraction & 6 \\
\hline Farine de luzerne déshydratée $\ldots \ldots \ldots \ldots \ldots \ldots \ldots \ldots \ldots \ldots$ & 6 \\
\hline Farine de viande $(\ldots, \ldots \ldots \ldots, \ldots \ldots \ldots \ldots \ldots \ldots \ldots \ldots$, & 5 \\
\hline Calcimarine $\ldots \ldots \ldots \ldots \ldots \ldots \ldots$ & 8 \\
\hline Complément minéral et vitaminique $\ldots$. & 2,4 \\
\hline D.L. méthionine $\ldots \ldots \ldots \ldots \ldots \ldots \ldots$ & 0,1 \\
\hline
\end{tabular}

\section{B. Méthodes d'analyse}

Les lipides totaux ont été extraits, puis purifiés à partir d'une fraction aliquote de chaque jaune d’ouf en utilisant le mélange chloroforme-méthanol $(2 / 1 ; \mathrm{v} / \mathrm{v})$ et selon la méthode proposée par Folch et al. (1957). Les teneurs en lipides totaux ont été déterminées par pesée après évaporation des solvants. Elles sont exprimées en pourcentage du poids de jaune d'œuf.

Les concentrations en phospholipides (PL) ont été obtenues par dosage du phosphore (P) dans les lipides totaux $(P L=P \times 25)$ selon la technique de BART- 
LETT (1959). Les valeurs sont exprimées en p. 100 des lipides totaux. Le cholestérol total (CT) a été dosé à partir d'une fraction aliquote de jaune en utilisant une méthode enzymatique faisant intervenir la cholestérol oxydase (EC 1.1.3.6.) et la catalase (EC 1.11.1.6.) (Boeringher ${ }^{\circledR}$, Mannheim, R.F.A.). Les valeurs obtenues, rapportées à la teneur en lipides totaux des échantillons, sont exprimées en p. 100 des lipides totaux.

Compte tenu de l'analyse des nombreux résultats disponibles dans la littérature, et en particulier ceux passés en revue par PARKInson (1966) il apparaît que les teneurs en triglycérides (TG) dans les lipides totaux de l'œuf peuvent théoriquement être estimées à partir des valeurs enregistrées pour les phospholipides et le cholestérol en utilisant l'équation suivante : TG $(\%)=100-[\mathrm{PL}(\%)+\mathrm{CT}(\%)]$. Les dosages enzymatiques (Kit Boeringher ${ }^{\circledR}$ ) réalisés sur 8 échantillons de jaunes pris au hasard, et provenant de chacun des 2 génotypes étudiés (4 échantillons par génotype), ont permis de confirmer qu'il n'existait pas de différences entre les valeurs ainsi déterminées et celles qui avaient été obtenues par le calcul. Pour le présent travail, on a donc considéré que les valeurs obtenues par le calcul représentaient une bonne estimation des teneurs en triglycérides des lipides totaux.

Les triglycérides et les phospholipides ont été séparés à partir du mélange de lipides totaux en utilisant la chromatographie préparative sur couche épaisse de gel de silice $G$. Une première migration a été réalisée à l'aide du mélange Hexane-Ether $(40 / 30 ; \mathrm{v} / \mathrm{v})$. Elle a été suivie d'une seconde migration réalisée en utilisant le mélange hexane-ether-méthanol-acide formique $(40-60-0,8-0,08)$ qui améliore la séparation entre les différentes classes de lipides et, en particulier, évite toute éventuelle interférence entre monoglycérides et phospholipides. Les bandes correspondant aux triglycérides et aux phospholipides ont été repérées par rapport au niveau de migration des substances pures servant de références. Les triglycérides et les phospholipides ont été récupérés par élution à partir du gel de silice.

Les acides gras des lipides totaux et des fractions purifiées de triglycérides ou de phospholipides ont été séparés par saponification. Ils ont ensuite été méthylés et analysés par chromatographie en phase gazeuse. Les conditions analytiques ont été rapportées antérieurement (DEMARNe et al., 1980). Les compositions en acides gras sont exprimées en pourcentage (en masse) de l'ensemble des esters méthyliques dosés.

\section{Analyses statistiques}

Toutes les comparaisons entre génotypes sont faites par test de Student-Fisher sur les différences entre individu normal et nain de chaque couple (méthode des couples).

\section{Résultats}

Le tableau 2 indique, pour la seconde série seule, le poids de l'œuf, le poids des jaunes et leur teneur en lipides totaux, triglycérides, phospholipides et cholestérol, pour chaque génotype. Le tableau 3 contient, pour cette série ainsi que pour la première et le total des deux, la composition des lipides totaux pour les acides gras principaux. Enfin, le détail de cette composition pour les triglycérides et phospholipides (6 couples de la $2^{\mathrm{e}}$ série) est donné au tableau 4. 


\section{TABLEaU 2}

Pourcentage des lipides totaux et des triglycérides, phospholipides et cholestérol des jaunes d'cufs de poules normales $(D w+)$ et naines $(d w)$

( $2^{e}$ série : $N=12$ couples).

Percentage of total lipids and triglycerides,

phospholipids and cholesterol in yolks from normal $(D w+)$ or dwarf $(d w)$ hens.

\begin{tabular}{|c|c|c|c|}
\hline \multirow{2}{*}{ Variable } & \multicolumn{2}{|c|}{ Valeur moyenne } & \multirow{2}{*}{$t$ et signification } \\
\hline & $\mathrm{Dw}+$ & $d w$ & \\
\hline Poids de l'œuf $(g) \ldots \ldots \ldots$ & $63,20 \pm 0,78$ & $56,47 \pm 1,92$ & $3,22 * *$ \\
\hline Poids du jaune $(\mathrm{g}) \ldots \ldots \ldots$ & $16,88 \pm 0,20$ & $15,27 \pm 0,51$ & $2,65^{*}$ \\
\hline Lipides totaux (p. 100) ..... & $32,01 \pm 0,59$ & $31,65 \pm 0,49$ & 0,49 \\
\hline $\begin{array}{r}\text { Triglycérides (p. } 100 \text { des lipi- } \\
\text { des totaux) } \ldots \ldots \ldots \ldots \ldots \ldots\end{array}$ & 67,95 & 66,76 & \\
\hline Phospholipides (p. 100) & $28,39 \pm 0,59$ & $29,68 \pm 0,58$ & 1,83 \\
\hline Cholestérol (p. 100) ........ & $3,66 \pm 0,11$ & $3,56 \pm 0,08$ & 0,69 \\
\hline
\end{tabular}

* Différence significative au seuil 5 p. 100 .

** Différence significative au seuil 1 p. 100. 


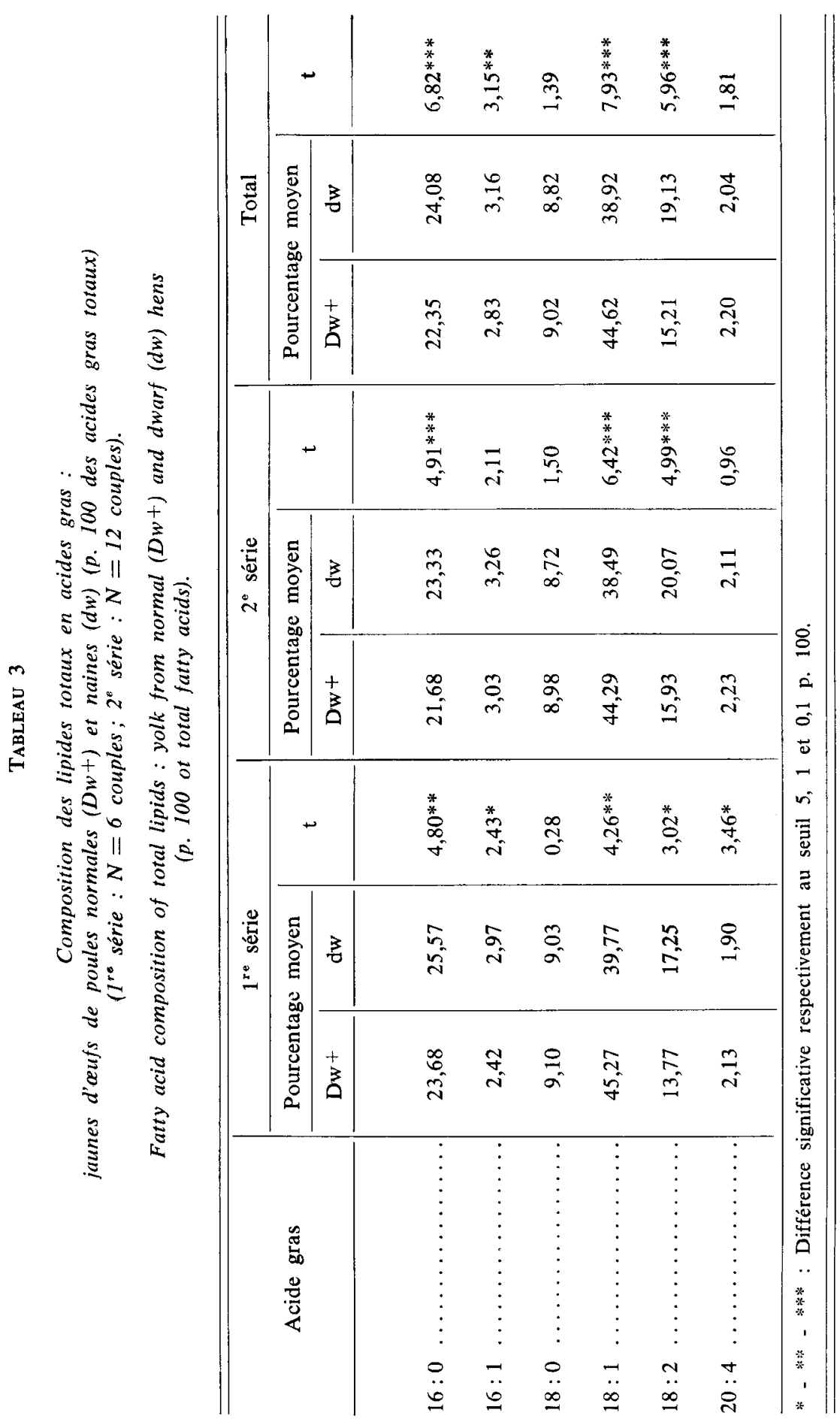




\section{TABLEAU 4}

Composition en acides gras des triglycérides et des phospholipides : jaunes d'aufs de poules normales ( $D w+$ ) et naines $(d w)$ ( $2^{\circ}$ série $: N=6$ couples) ( $p .100$ des acides gras totaux).

Fatty acid composition of triglycerides and phospholipids : yolks from normal $(\mathrm{Dw}+)$ and dwarf (dw) hens (p. 100 of total fatty acids).

\begin{tabular}{|c|c|c|c|c|c|c|c|}
\hline & \multirow{3}{*}{ Acide gras } & \multicolumn{3}{|c|}{ Triglycérides } & \multicolumn{3}{|c|}{ Phospholipides } \\
\hline & & \multicolumn{2}{|c|}{ Pourcentage moyen } & \multirow{2}{*}{$\mathrm{t}$} & \multicolumn{2}{|c|}{ Pourcentage moyen } & \multirow{2}{*}{$\mathbf{t}$} \\
\hline & & $\mathrm{Dw}+$ & $d w$ & & $\mathrm{Dw}+$ & $d w$ & \\
\hline $16: 0$ & & 23,42 & 24,72 & 2,09 & 24,02 & 25,02 & 1,18 \\
\hline $16: 1$ & & 2,85 & 3,52 & $2,85^{*}$ & 一(1) & 一(1) & 一 \\
\hline $18: 0$ & & 7,33 & 5,57 & $5,88^{* *}$ & 21,70 & 21,30 & 0,33 \\
\hline $18: 1$ & & 51,78 & 43,33 & $7,04 * * *$ & 25,63 & 23,20 & 1,87 \\
\hline $18: 2$ & & 14,78 & 22,87 & $7,61 * * *$ & 16,90 & 18,93 & 2,16 \\
\hline $20: 4$ & & 一 & 一 & - & 12,08 & 11,55 & 0,55 \\
\hline
\end{tabular}

$*_{-} * *_{-} * * *$ : Différence significative respectivement au seuil 5,1 et 0,1 p. 100 .

(1) $<1$ p. 100.

Le poids moyen des jaunes est inférieur pour les poules naines. Par contre, ni la teneur du jaune en lipides totaux, ni la répartition de ces lipides en triglycérides, phospholipides et cholestérol ne diffèrent significativement suivant le génotype au locus Dw (tableau 2).

Le tableau 3 révèle entre œufs de poules naines et normales des différences très hautement significatives pour la composition en acides gras dans chaque série et sur le total. Ceci est particulièrement marqué pour les acides $18: 1$ (oléique) et $18: 2$ (linoléique), le second étant plus important et le premier moins important en pourcentage dans l'œuf de la pondeuse naine. Si l'on considère le rapport du pourcentage d'acide oléique à celui de l'acide linoléique, non donné dans le tableau, il est voisin de 3 et de 2 respectivement pour les génotypes $\mathrm{Dw}^{+}$et dw. Les taux des acides 16:0 (palmitique) et $16: 1$ (palmitoléique) diffèrent dans une proportion moins importante mais cependant significative (plus de 16:1 et de 16:0 dans l'œuf des poules naines).

Enfin, le tableau 4 montre que les différences ci-dessus proviennent exclusivement des triglycérides. Aucun pourcentage concernant les phospholipides ne diffère significativement selon le génotype. Par contre, pour les triglycérides, l'écart entre naines et normales pour la teneur respective en acide oléique et linéoléique est encore accentué par comparaison avec les lipides totaux, la teneur en acide oléique étant d'environ 8 p. 100 plus faible, et celle de l'acide linoléique de 8 p. 100 plus élevée 
pour les poules naines que pour les poules normales. Une différence non apparente au tableau 3 se révèle en outre cette fois pour l'acide 18:0 (stéarique), dont le taux est abaissé dans l'œuf des naines.

\section{Discussion}

L'étude comparative des lipides de l'œuf chez les poules Leghorn normales ou naines ne met donc pas en évidence de différences significatives pour ce qui concerne les teneurs en lipides totaux et la répartition des différentes classes de lipides. De plus, les valeurs présentées dans ce travail sont similaires à celles rapportées antérieurement pour des œufs provenant d'autres souches (PARKInson, 1966). Les seules différences significatives apparaissent au niveau de la composition en acides gras des triglycérides et se traduisent surtout par une teneur plus élevée en acide linoléique $(18: 2 n-6)$ et plus faible en acide oléique (18:1n-9) dans les æufs de poules du génotype dw par rapport au génotype $\mathrm{Dw}^{\dagger}$. A notre connaissance, cette différence entre les deux génotypes n'a jamais été décrite dans la littérature.

Il s'agit d'un premier résultat, et d'autres données seront nécessaires pour préciser une interprétation. En particulier, les estimations de l'ingestion et de l'exportation des lipides devront être précisées. Cependant, une hypothèse provisoire peut être émise. Les acides gras estérifiés dans les lipides de l'œuf proviennent de trois sources : l'aliment (lipides exogènes), la synthèse hépatique (lipides endogènes) et l'hydrolyse des triglycérides des réserves adipeuses, fournissant les acides gras libres plasmatiques (LECLERCQ, 1973). L'acide linoléique, à la différence de l'acide oléique, ne peut pas être synthétisé par l'animal, et son origine est donc exclusivement exogène (BIERI et al., 1956; Hopkins \& Nesheim, 1967). On a rapporté antérieurement que les teneurs en acides gras libres au niveau du plasma sont significativement moins élevées chez la poule naine que chez la poule normale (Guillaume, 1975). Dans ces conditions, l'accroissement des teneurs en acide linoléique dans l'œuf pour le génotype dw pourrait être le reflet d'une participation accrue des lipides alimentaires lors de la synthèse des triglycérides vitellins chez la poule naine. Cependant, et compte tenu des résultats dont on dispose à l'heure actuelle, on ne peut pas écarter l'éventualité d'une différence de composition en acides gras des triglycérides de réserve entre les deux génotypes. Si celle-ci se traduit par une teneur plus élevée en acide linoléique $(18: 2 n-6)$ pour le génotype $d w$, ceci pourrait également expliquer pour le moins partiellement, les différences observées. Des études complémentaires, tant biochimiques que nutritionnelles, sont envisagées afin de rechercher l'origine de ces variations liées au gène $\mathrm{dw}$.

Reçu le 20 avril 1983.

Accepté le 15 novembre 1983.

\section{Références bibliographiques}

Banerjee A.K., Bordas A., Merat P., 1982. Sex-linked dwarf gene (dw) in White Leghorn laying hens under normal or hot temperature. Ann. Génét. Sél. Anim., 14, 135-160. 
Banerjee A.K., Merat P., Bordas A., 1982. Egg yolk production traits in relation to ovum development, liver and liver moisture weight in dwarf and normal White Leghorns. Ann. Génét. Sél. Anim., 14, 369-380.

Bartlett G.R., 1959. Phosphorus assay in column chromatography. J. Biol. Chem., 234, 466-468.

Bieri J.G., Briggs G.M., Spivey Fox M.R., Pollard C.J., Ortiz L.O., 1956. Essential fatty acids in the chick. I. - Development of fat deficiency. Proc. Soc. Exp. Biol. Med., 93, $237-240$.

Calabotta D.F., Cherry J.A., Siegel P.B., 1982. Lipogenesis and lipolysis in normal dwarf males of lines selected for high and low body weight. Poult. Sci., 61, 1130-1131 (abstr.).

Demarne Y., Thouard D., Pihet A., Lecourtier M.J., 1980. Effect of different levels of dietary fats upon the positional distribution of fatty acids in rat body triacylglycerols. Comp. Biochem. Physiol., 68 A, 361-371.

Folch J., Lees M., Sloane-Stanley G.H., 1957. A simple method for the isolation and purification of total lipids from animal tissues. J. Biol. Chem., 226, 497-509.

Guillaume J., 1969. Conséquences de l'introduction du gène de nanisme dw sur l'utilisation alimentaire chez le poussin femelle. Ann. Biol. Anim. Bioch. Biophys., 9, 369-378.

Guillaume J., 1975. Recherches sur les caractéristiques nutritionnelles et métaboliques du poussin nain $d w$. Thèse docteur ès sciences, Tours.

Guillaume J., 1976. The dwarfing gene dw : its effects on anatomy, physiology, nutrition, management. Its application in Poultry industry. World's Poult. Sci. J., 32, 285-304.

Hopkins D.T., Nesheim M.C., 1967. The linoleic acid requirement of chicks. Poult. Sci.. 46, $872-881$.

JAAP R.G., 1968. Reproductive idiosyncrasies of the broiler pullet. C.R. $3^{\circ}$ Conférence Avicole Européenne, Jérusalem, 8-12 septembre 1968, 74-79.

JAAP R.G., 1969. Sex-linked dwarfism and egg production of broiler dams. Poult. Sci., 48, 344-346.

LECLERCQ B., 1973. Contribution de l'aliment et des réserves corporelles à la genèse des lipides vitellins de la poule. Thèse doctorat ès sciences, Paris.

Merat P., 1972. Quelques effets du gène dw sur la ponte et sur la qualité des œufs. Ann. Génét. Sél. Anim., 4, 217-223.

Merat P., Guillaume J., 1969. Etude d'un gène du nanisme lié au sexe chez la poule. II. - Fonctionnement thyroïdien. Ann. Génét. Sél. Anim., 1, 131-133.

Merat P., Ricard F.H., 1974. Etude d'un gène de nanisme lié au sexe chez la poule. Importance de l'état d'engraissement et gain de poids chez l'adulte. Ann. Génét. Sél. Anim., 6, 211-218.

VAN Middelkoop M., 1973. Influence of the dwarfing gene on yolk production and its consequences for normal egg laying of White Plymouth Rock pullets. Arch. Geflügelkd., 37, 192-196.

Ouhayoun J., 1970. Etude d'un gène de nanisme lié au sexe chez la poule. V. - Composition corporelle in vivo. Ann. Génét. Sél. Anim., 2, 33-36.

Parkinson T.L., 1966. The chemical composition of eggs. J. Sci. food Agric., 17, 101-111.

Polkinghorne R.W., 1974. Fat content of birds possessing the Para Minigene. J. Austr. Inst. Agric. Sci., 40, 74-75.

RiCARD F.H., 1970. Etude d'un gène du nanisme lié au sexe chez la poule. IV. - Observations sur la croissance et les caractéristiques de carcasse du jeune poulet. Ann. Génét. Sél. Anim., 2, 19-31.

Touchburn S.P., Blum J.C., 1972. Effects of the genes for dwarfism (dw) and naked neck (Na) on chick growth and lipid metabolism. Ann. Génét. Sél. Anim., 4, 311-316.

Washburn K.W., 1979. Genetic variation in the chemical composition of the egg. Poult. Sci., 58, 529-535. 